\title{
Advanced Multipath Mitigation Techniques for Satellite-Based Positioning Applications
}

\author{
Mohammad Zahidul H. Bhuiyan and Elena Simona Lohan \\ Department of Communications Engineering, Tampere University of Technology, P.O. Box 553, 33101 Tampere, Finland \\ Correspondence should be addressed to Mohammad Zahidul H. Bhuiyan, mohammad.bhuiyan@tut.fi
}

Received 9 June 2010; Revised 17 August 2010; Accepted 20 October 2010

Academic Editor: Olivier Julien

Copyright ( $) 2010$ M. Z. H. Bhuiyan and E. S. Lohan. This is an open access article distributed under the Creative Commons Attribution License, which permits unrestricted use, distribution, and reproduction in any medium, provided the original work is properly cited.

\begin{abstract}
Multipath remains a dominant source of ranging errors in Global Navigation Satellite Systems (GNSS), such as the Global Positioning System (GPS) or the future European satellite navigation system Galileo. Multipath is generally considered undesirable in the context of GNSS, since the reception of multipath can make significant distortion to the shape of the correlation function used for time delay estimation. However, some wireless communications techniques exploit multipath in order to provide signal diversity though in GNSS, the major challenge is to effectively mitigate the multipath, since we are interested only in the satellitereceiver transit time offset of the Line-Of-Sight (LOS) signal for the receiver's position estimate. Therefore, the multipath problem has been approached from several directions in order to mitigate the impact of multipath on navigation receivers, including the development of novel signal processing techniques. In this paper, we propose a maximum likelihood-based technique, namely, the Reduced Search Space Maximum Likelihood (RSSML) delay estimator, which is capable of mitigating the multipath effects reasonably well at the expense of increased complexity. The proposed RSSML attempts to compensate the multipath error contribution by performing a nonlinear curve fit on the input correlation function, which finds a perfect match from a set of ideal reference correlation functions with certain amplitude(s), phase(s), and delay(s) of the multipath signal. It also incorporates a threshold-based peak detection method, which eventually reduces the code-delay search space significantly. However, the downfall of RSSML is the memory requirement which it uses to store the reference correlation functions. The multipath performance of other delay-tracking methods previously studied for Binary Phase Shift Keying-(BPSK-) and Sine Binary Offset Carrier- (SinBOC-) modulated signals is also analyzed in closed loop model with the new Composite BOC (CBOC) modulation chosen for Galileo E1 signal. The simulation results show that the RSSML achieves the best multipath mitigation performance in a uniformly distributed two-to-four paths Rayleigh fading channel model for all three modulated signals.
\end{abstract}

\section{Introduction}

Multipath remains a dominant source of ranging errors in Global Navigation Satellite Systems (GNSSs), such as the Global Positioning System (GPS) or the future European satellite navigation system, Galileo. Several approaches have been used in order to reduce the multipath error. Among them, the use of special multipath-limiting antennas (i.e., choke ring or multibeam antennas), the postprocessing techniques to reduce carrier multipath, the carrier smoothing to reduce code multipath, and the code-tracking algorithms based on receiver internal correlation technique are the most prominent approaches [1]. In this paper, our focus is limited to the correlation-based multipath mitigation techniques, since they are the most widely used in commercial GNSS receivers. The classical correlation-based code tracking structure used in GNSS is based on a feedback delay estimator and is implemented via a feedback loop. The most known feedback-delay estimator is the Delay Lock Loop (DLL) or Early-Minus-Late (EML) loop, where two correlators spaced at one chip from each other are used in the receiver in order to form a discriminator function, whose zero crossings determine the path delays of the received signal [2-7]. The classical EML fails to cope with multipath propagation [1]. Therefore, several enhanced EML-based techniques have been introduced in the literature for the last two decades in order to mitigate the impact of multipath, especially in closely spaced path scenarios. One class of these enhanced 
EML techniques is based on the idea of narrowing the spacing between the early and late correlators, that is, narrow EML (nEML) or narrow correlator [1, 8, 9]. The choice of correlator spacing depends on the receiver's available frontend bandwidth along with the associated sampling frequency [10]. Correlator spacings in the range of 0.05 to 0.2 chips are commercially available for nEML-based GPS receivers [11].

Another family of discriminator-based DLL variants proposed for GNSS is the so-called Double-Delta $(\Delta \Delta)$ technique, which uses more than 3 correlators in the tracking loop (typically, 5 correlators: two early, one in prompt and two late) [8]. $\Delta \Delta$ technique offers better multipath rejection in medium-to-long delay multipath $[9,12]$ in good Carrier-to-Noise-density ratio $\left(C / N_{0}\right)$. Couple of wellknown particular cases of $\Delta \Delta$ technique are the High Resolution Correlator (HRC) [9], the Strobe Correlator (SC) $[8,13]$, the Pulse Aperture Correlator (PAC) [14], and the modified correlator reference waveform [8, 15]. One other similar tracking structure is the Multiple Gate Delay (MGD) correlator [16-19], where the number of early and late gates and the weighting factors used to combine them in the discriminator are the parameters of the model and can be optimized according to the multipath profile as illustrated in [12]. While coping better with the ambiguities of BOC correlation function, the MGD provides slightly better performance than the nEML at the expense of higher complexity and is sensitive to the parameters chosen in the discriminator function (i.e., weights, number of correlators, and correlator spacing) $[12,19]$. In [12], it is also shown that $\Delta \Delta$ technique is a particular case of MGD implementation.

Another tracking structure closely related to $\Delta \Delta$ technique is the Early1/Early2 (E1/E2) tracker, initially proposed in [20] and later described in [8]. In E1/E2 tracker, the main purpose is to find a tracking point on the correlation function that is not distorted by multipath. The first step is to locate two correlators on the early slope of the correlation function. The correlation values of these two early correlators are then compared with the correlation values of an ideal reference correlation function. Finally, a delay-correction factor is computed based on the measured and reference correlation values of E1 and E2 correlators. As reported in [8], E1/E2 tracker shows some performance improvement over $\Delta \Delta$ technique only for very short delay multipath for GPS L1 C/A signal (i.e., BPSK signal).

Another feedback-tracking structure is the Early-LateSlope (ELS) [8], which is also known as Multipath Elimination Technique (MET) [21]. The ELS is based on two correlator pairs at both sides of the correlation function's central peak with parameterized spacing. Once both slopes are known, they can be used to compute a pseudorange correction that can be applied to the pseudorange measurement. However, simulation results performed in [8] showed that ELS is outperformed by HRC with respect to Multipath Error Envelopes (MEEs), for both BPSK and $\operatorname{SinBOC}(1,1)$ modulated signals.

A new multipath-estimation technique, named as APosteriori Multipath Estimation (APME), is proposed in [22], which relies on a posteriori estimation of the multipath error tracking. Multipath error is estimated independently in a multipath-estimator module on the basis of the correlation values from the prompt and very late correlators. The performance in multipath environment reported in [22] is comparable with that of the SC: slight improvement for very short delays (i.e., delays less than 20 meters), but rather significant deterioration for medium delays. A similar slopebased multipath mitigation strategy, named as Slope-based Multipath Estimator (SBME), was proposed by the authors in [23]. SBME first derives a multipath estimation equation by utilizing the correlation shape of the ideal normalized correlation function, which is then used to compensate for the multipath bias of an nEML tracking loop. SBME requires an additional correlator at the late side of the correlation function, and it is used in-conjunction with an nEML tracking loop. It is reported in [23] that SBME has superior multipath mitigation performance than nEML in closely spaced two paths channel model.

The conventional techniques, discussed so far can be classified based on their correlator requirements as shown in Figure 1. For clarity reason, we use the notation correlator in this paper in order to represent complex correlator (i.e., one complex correlator is equivalent to two correlators needed for in-phase and quad-phase channels).

One of the most promising advanced multipath mitigation techniques is the Multipath Estimating Delay Lock Loop (MEDLL) [24-26] implemented by NovAtel for GPS receivers. The MEDLL uses many correlators in order to determine accurately the shape of the multipath corrupted correlation function. Then, a reference function is used in a software module in order to determine the best combination of LOS and Non-LOS (NLOS) components (i.e., amplitudes, delays, phases, and number of multipath). However, MEDLL provides superior long-delay multipath mitigation performance than $\mathrm{nEML}$ at the cost of expensive multicorrelatorbased tracking structure. MEDLL is considered as a significant evolutionary step in the receiver-based attempt to mitigate-multipath. Moreover, MEDLL has stimulated the design of different maximum likelihood-based implementations for multipath mitigation. One such variant is the noncoherent MEDLL, developed by the authors, as described in [27]. Classical MEDLL is based on a maximum likelihood search, which is computationally extensive. The authors implemented a noncoherent version of MEDLL that reduces the search space by incorporating a phase-search unit, based on statistical distribution of multipath phases. However, the performance of this suggested approach depends on the number of random phases considered; this means that the larger the number is, the better the performance will be. But this will also increase the processing burden significantly. The results reported in [27] show that the noncoherent MEDLL provides very good performance in terms of Root-MeanSquare-Error (RMSE), but has a rather poor Mean-Timeto-Lose-Lock (MTLL) as compared to the conventional DLL techniques.

A new technique to mitigate multipath by means of correlator reference waveform was proposed in [28]. This technique, referred to as second derivative correlator, generates a signal correlation function which has a much narrower width than a standard correlation function and 


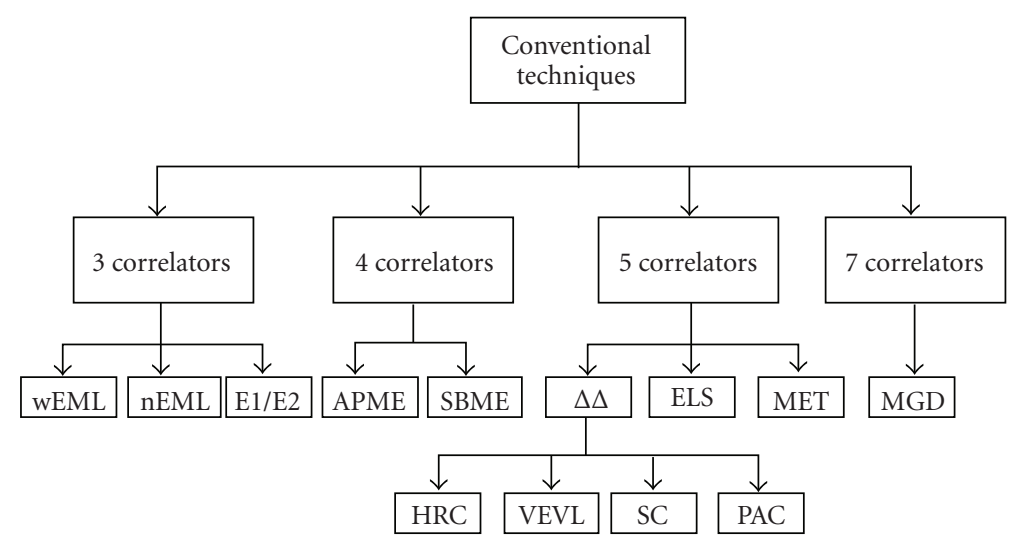

FIGURE 1: Classification of conventional multipath mitigation techniques based on correlator requirement.

is, therefore, capable of mitigating multipath errors over a much wider range of secondary path delays. The narrowing of correlation function is accomplished by using a specially designed code reference waveform (i.e., the negative of the second-order derivative of correlation function) instead of the ideal code waveform used in almost all existing receivers. However, this new technique reduces the multipath errors at the expense of a moderate decrease in the effective Signalto-Noise Ratio (SNR) due to the effect of narrowing the correlation function. A similar strategy, named as Slope Differential (SD), is based on second order derivative of the correlation function [29]. It is shown in [29] that this technique has better multipath performance than nEML and Strobe Correlator. However, the performance measure was solely based on the theoretical MEE curves, thus its potential benefit in more realistic multipath environment is still an open issue.

A completely different approach to mitigate multipath error is used in NovAtel's recently developed vision correlator [30]. The Vision Correlator (VC) is based on the concept of Multipath Mitigation Technique (MMT) developed in [31]. It can provide a significant improvement in detecting and removing multipath signals as compared to other standard multipath-resistant code-tracking algorithms (e.g., PAC of NovAtel). However, VC has the shortcoming that it requires a reference function shape to be used to fit the incoming data with the direct path and the secondary path reference signals. The reference function generation has to be accomplished a priori, and it must incorporate the issues related to Radio Frequency (RF) distortions introduced by RF front end.

Several advanced multipath mitigation techniques were also proposed by the authors in $[27,32]$. While improving the delay-estimation accuracy, these techniques require a higher number of correlators than the traditional DLL, and they are sensitive to the noise-dependent threshold choice. Among these advanced techniques, two most competitive ones, previously proposed by the authors, are selected herein for performance comparison. These are Peak Tracking, based on 2nd-order Differentiation (PT(Diff2)), and TeagerKaiser- (TK-) based delay estimation, the details of which can be found in [27].
Many correlation-based multipath mitigation techniques exist, but even the most promising ones (e.g., nEML, HRC, PT(Diff2), etc.) are not good enough for closely spaced multipath environment, which is a key motivation for presentday researchers (as is the case in this research) to come up with new innovative techniques. The purpose of this paper is twofold: first, to propose a novel maximum likelihood-based Reduced Search Space Maximum Likelihood (RSSML) delay estimator as an advanced multipath mitigation technique, mostly designed for harsh multipath environment (where there can be more than two strong closely spaced paths) and second, to analyze the performance of other contemporary multipath mitigation techniques (both conventional and advanced techniques) under the same unified simulation model. Additionally, the authors also develop a combined TK- and nEML-based approach, named here as TK+nEML, which is less complex than TK, while at the same time provide better multipath mitigation than nEML. The motivation for such a combined approach will be discussed in more detail in Section 4.2. The authors remark here that the basic idea of RSSML was first introduced in [33], where RSSML was implemented for two paths channel with infinite bandwidth assumption. Moreover, the version of RSSML presented in [33] was not optimized in terms of memory, since it required a large set of correlation functions for all possible delays in a predefined code delay window range. The RSSML with its current version requires a large set of correlation functions only for the prompt correlator, and it is also adapted for finite bandwidth assumption for any number of paths up to four. Simulation results in fading multipath environment are included in this paper in order to compare the performance of the proposed techniques with the various conventional DLLs and other developed advanced techniques (which are briefly reviewed here). The performance of these techniques are analyzed for the newly defined Composite Binary Offset Carrier (CBOC) modulation along with the existing Binary Phase Shift Keying (BPSK) and Sine Binary Offset Carrier (SinBOC) modulations.

The rest of this paper is organized as follows. Section 2 presents the signal and channel model, followed by a description on multicorrelator-based delay-tracking structure in 
Section 3. The advanced multipath mitigation techniques including the proposed RSSML are introduced in Section 4, followed by a detailed analysis on implementation issues for RSSML in Section 5. Section 6 shows the multipath performance of the selected techniques in terms of semianalytical running average error. Section 7 presents the simulation results in two-to-four paths fading channel model with finite front-end bandwidth whereas Section 8 provides a comparison between different techniques in terms of their multipath mitigation capability, relative complexity and needed a priori information. Finally, some general conclusions are drawn in Section 9, with a perspective on future research direction.

\section{Signal and Channel Model}

Typical GNSS signals, such as those used in GPS or Galileo, employ the Direct Sequence-Code Division Multiple Access (DS-CDMA) technique, where a Pseudorandom Noise (PRN) code from a specific satellite is spreading the navigation data over $S_{F}$ chips (or over a code-epoch length) $[34,35]$. In what follows, a baseband model is adopted for clarity reason. The estimation of code delay in today's receivers is typically done in digital domain using the baseband correlation samples. In the following, the time notation $t$ denotes the discrete time instant. The signal $x(t)$ transmitted from one satellite with a specific PRN code can be written as

$$
x(t)=\sqrt{E_{b}} p_{\bmod }(t) \otimes c(t)
$$

where $E_{b}$ is the bit energy, $p_{\bmod }(t)$ is the modulation waveform (i.e., BPSK for GPS L1 C/A code or CBOC(-) for Galileo E1C signals), and $c(t)$ is the navigation data after spreading as written below (spreading is done with a PRN code of chip interval $T_{c}$ and spreading factor $S_{F}$ )

$$
c(t)=\sum_{n=-\infty}^{\infty} b_{n} \sum_{k=1}^{S_{F}} c_{k, n} \delta\left(t-n T_{c} S_{F}-k T_{c}\right)
$$

Above $\delta(\cdot)$ is the Dirac unit pulse, $b_{n}$ is the $n$th data bit (for pilot channels, $\left.b_{n}=1, \forall n\right)$, and $c_{k, n}$ is the $k$ th chip $( \pm 1$ valued) corresponding to the $n$th spread bit.

The modulation waveform for BPSK or BOC can be written as [36]

$$
p_{\text {mod }}(t)=p_{T_{B}}(t) \otimes \sum_{i=0}^{N_{B}-1} \delta\left(t-i T_{B}\right)
$$

where $N_{B}$ is BOC modulation order: $N_{B}=1$ for BPSK modulation (BPSK can be seen as a particular case of BOC modulation, as illustrated in [36]) and $N_{B}=2 f_{s c} / f_{c}$, where $f_{s c}$ is the subcarrier frequency and $f_{c}$ is the carrier frequency for BOC modulation, $T_{B}=T_{c} / N_{B}$ is the BOC interval, and $p_{T_{B}}(t)$ is the pulse-shaping filter (e.g., for unlimited bandwidth case, $p_{T_{B}}(t)$ is a rectangular pulse of width $T_{B}$ and unit amplitude).

The signal $x(t)$ is typically transmitted over a multipath static or fading channel, where all interference sources except the multipath are lumped into a single additive Gaussian noise term $\eta(t)$

$$
r(t)=\sum_{l=1}^{L} \alpha_{l} x\left(t-\tau_{l}\right) e^{j\left(2 \pi f_{D} t+\theta_{l}\right)}+\eta(t),
$$

where $r(t)$ is the received signal, $L$ is the number of channel paths, $\alpha_{l}$ is the amplitude of the $l$ th path, $\theta_{l}$ is the phase of the $l$ th path, $\tau_{l}$ is the channel delay introduced by the $l$ th path (typically assumed to be slowly varying or constant within the observation interval), $f_{D}$ is the Doppler shift introduced by the channel, and $\eta(t)$ is a wideband additive noise, incorporating all sources of interferences over the channel. Assuming that the signal is sampled at $N_{s}$ samples per-chip (for BPSK) or per-BOC interval (for BOC modulation), then the power spectral density of $\eta(\cdot)$ can be written as $N_{0} /\left(N_{s} N_{B} S_{F}\right)$, where $N_{0}$ is the noise power in $1 \mathrm{kHz}$ bandwidth (i.e., bandwidth corresponding to one code epoch). Generally, the SNR for any GNSS signal is expressed with respect to the code epoch bandwidth $B_{w}$, under the name of Carrier-to-Noise-density ratio $\left(C / N_{0}\right)$. The relationship between $\left(C / N_{0}\right)$ and bit-energy-to-noise ratio (in $\mathrm{dB}$ ) can be written as [37]

$$
\frac{C}{N_{0}}[\mathrm{~dB}-\mathrm{Hz}]=\frac{E_{b}}{N_{0}}+10 \log _{10}\left(B_{w}\right) .
$$

The delay tracking is typically based on the code epochby-epoch correlation $\mathcal{R}(\cdot)$ between the incoming signal and the reference $x_{\text {ref }}(\cdot)$ modulated PRN code, with a certain candidate Doppler frequency $\hat{f}_{D}$ and delay $\hat{\tau}$

$$
\mathcal{R}\left(\hat{\tau}, \hat{f}_{D}, m\right)=\mathbf{E}\left(\frac{1}{T_{\text {sym }}} \int_{(m-1) T_{\text {sym }}}^{m T_{\text {sym }}} r(t) x_{\text {ref }}\left(\hat{\tau}, \hat{f}_{D}\right) d t\right),
$$

where $m$ is the code epoch index, $T_{\text {sym }}$ is the symbol period (i.e., $T_{\text {sym }}=S_{F} T_{c}$ ), and $\mathrm{E}(\cdot)$ is the expectation operator with respect to the random variables (e.g., PRN code, channel effects, etc.), and

$$
\begin{aligned}
x_{\text {ref }}\left(\hat{\tau}, \hat{f}_{D}\right)= & p_{\text {mod }}(t) \otimes \sum_{n=-\infty}^{\infty} \sum_{k=1}^{S_{F}} \hat{b}_{n} c_{k, n} \\
& \times \delta\left(t-n T_{\text {sym }}-k T_{c}\right) \otimes p_{T_{B}}(t) e^{-j 2 \pi \hat{f}_{D} t},
\end{aligned}
$$

where $\hat{b}_{n}$ is the estimated data bits. For Galileo signals, a separate pilot channel is transmitted [34]. In what follows, it is assumed that data bits are perfectly estimated $\left(\hat{b}_{n}=\right.$ $b_{n}$ ), and removed before the correlation process. In a practical receiver, in order to cope with noise, coherent and noncoherent integration can be used. The average coherent correlation function $\overline{\mathcal{R}}_{c}\left(\hat{\tau}, \hat{f}_{D}\right)$ can be written as

$$
\overline{\mathcal{R}}_{c}\left(\hat{\tau}, \hat{f}_{D}\right)=\frac{1}{N_{c}} \sum_{m=1}^{N_{c}} \mathcal{R}\left(\hat{\tau}, \hat{f}_{D}, m\right)
$$

where $N_{c}$ is the coherent integration time expressed in code epochs or milliseconds for GPS or Galileo signal, and the 


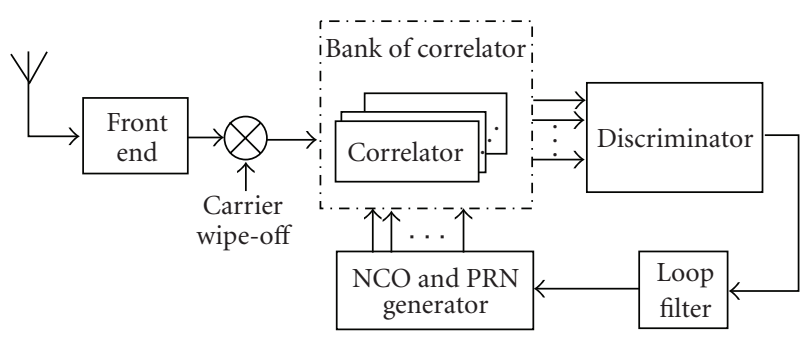

FIGURE 2: Block diagram for multicorrelator based DLL implementation.

noncoherently averaged correlation function $\overline{\mathcal{R}}_{\mathrm{nc}}\left(\hat{\tau}, \hat{f}_{D}\right)$ can be written as

$$
\overline{\mathcal{R}}_{\mathrm{nc}}\left(\hat{\tau}, \hat{f}_{D}\right)=\frac{1}{N_{\mathrm{nc}}} \sum_{N_{n c}}\left|\frac{1}{N_{c}} \sum_{m=1}^{N_{c}} \mathcal{R}\left(\hat{\tau}, \hat{f}_{D}, m\right)\right|^{p_{n c}},
$$

where $N_{\mathrm{nc}}$ is the noncoherent integration time expressed in blocks of length $N_{c}$ milliseconds (for clarity reason, we avoid using the block indexes for the noncoherent summations), and $p_{\mathrm{nc}}$ is the power index used for noncoherent summation. The most encountered variants for $p_{\mathrm{nc}}$ are: $p_{\mathrm{nc}}=1$ (which is the sum of absolute correlation values), and $p_{\mathrm{nc}}=2$ (i.e., which is the sum of squared-absolute correlation values). We prefer to use the later option (i.e., $p_{\mathrm{nc}}=2$ ) in our simulations.

\section{Multicorrelator Based Delay-Tracking Structure}

Compared with the conventional EML tracking loop, where only three correlators are used (i.e., Early, Prompt and Late), here, in the multicorrelator-based structure, we generate a bank of correlators (e.g., in this implementation, we use 193 correlators with 0.0208 chips spacing between successive correlators) as presented in Figure 2. This large number of correlators is needed in order to include the advanced multipath mitigation techniques in the comparison, because these techniques make use of these correlators for estimating the channel properties while taking decision about the code delay [27]. Some of these correlators can be kept inactive or unused, for example when EML and HRC tracking loops are used. After the necessary front-end processing, and after the carrier has wipedoff, the received postprocessed signal was passed through a bank of correlators. As shown in Figure 2, the NCO and PRN generator block produces a bank of early and late versions of replica codes based on the delay of the LOS signal $\hat{\tau}$, the correlator spacing $\Delta$, and the number of correlators $M$. In case of EML-tracking loop, the corresponding early-late spacing is equal to $2 \Delta$. The received signal is correlated with each replica in the correlator bank, and the output of the correlator bank is a vector of samples in the correlation envelope. Therefore, we obtain the correlation values for the range of $\pm M \Delta$ chips from the prompt correlator, where $M$ is the number of correlators and $\Delta$ is the correlator spacing between successive correlators. The various code tracking techniques (named as discriminator in Figure 2) utilize the correlation values as input, and generate the estimated LOS delay as output, which is then smoothed by a loop filter. In accordance with [35], the implemented code loop filter is a 1st order filter, whose function can be written as

$$
\widehat{\tau}(k+1)=\hat{\tau}(k)+\gamma d(k),
$$

where $\gamma$ is calculated based on loop filter bandwidth, $B_{n}$. A DLL loop bandwidth of $2 \mathrm{~Hz}$ is used in the simulation, assuming that carrier aiding is always available [9].

\section{Advanced Multipath Mitigation Techniques}

The advanced state-of-the-art multipath-mitigation techniques discussed in Section 1 are classified here based on their mitigation strategies, as shown in Figure 3. These advanced techniques usually require a vast number of correlators in order to estimate the channel characteristics, which are then used to mitigate the multipath effect. Several multipath-mitigation techniques introduced in past years are based on Maximum Likelihood (ML) estimation principle. Examples of ML-based techniques include MEDLL [26], MMT [31], VC [30] of NovAtel, MEDLL of Tampere University of Technology (TUT) [27], and the proposed RSSML. Among other techniques, second derivative [28], slope differential [29], and PT(Diff2) [27] are based on $2^{\text {nd }}$-order differentiation whereas TK, PT(TK) and TK + nEML are based on Teager Kaiser operator. In the following subsections, only those algorithms are elaborated, which will later be considered for performance analysis. It is nice to mention here that a brief discussion of the remaining algorithms has already been presented in Section 1 .

4.1. Teager Kaiser. The Teager Kaiser-based delay-estimation technique is based on the principle of extracting the signal energy corresponding to various channel paths via the nonlinear TK operator. The output $\Psi_{\mathrm{TK}}(x(n))$ of TK operator applied to a discrete signal $x(n)$, can be defined as [38]

$$
\begin{aligned}
\Psi_{\mathrm{TK}}(x(n))= & x(n-1) x^{*}(n-1) \\
& -\frac{1}{2}\left[x(n-2) x^{*}(n)+x(n) x^{*}(n-2)\right] .
\end{aligned}
$$

The input of TK operator can be the noncoherent correlation function. The output of TK operator can indicate the presence of a multipath component more clearly than looking directly at the correlation function. According to (11), at least 3 correlation values are needed to compute TK (in prompt, early, and very early). But usually, TK-based delay estimation utilizes a higher number of correlators and is sensitive to the noise dependent threshold choice. Firstly, it computes the noise variance according to the explanation presented in Section 5.1, which is then used to compute an adaptive threshold as defined in [27]. The peaks which are above the adaptive threshold are considered as competitive peaks. Among all the competitive peaks, TK selects the delay associated to that competitive peak which has the closest delay difference from the previous delay estimate. 


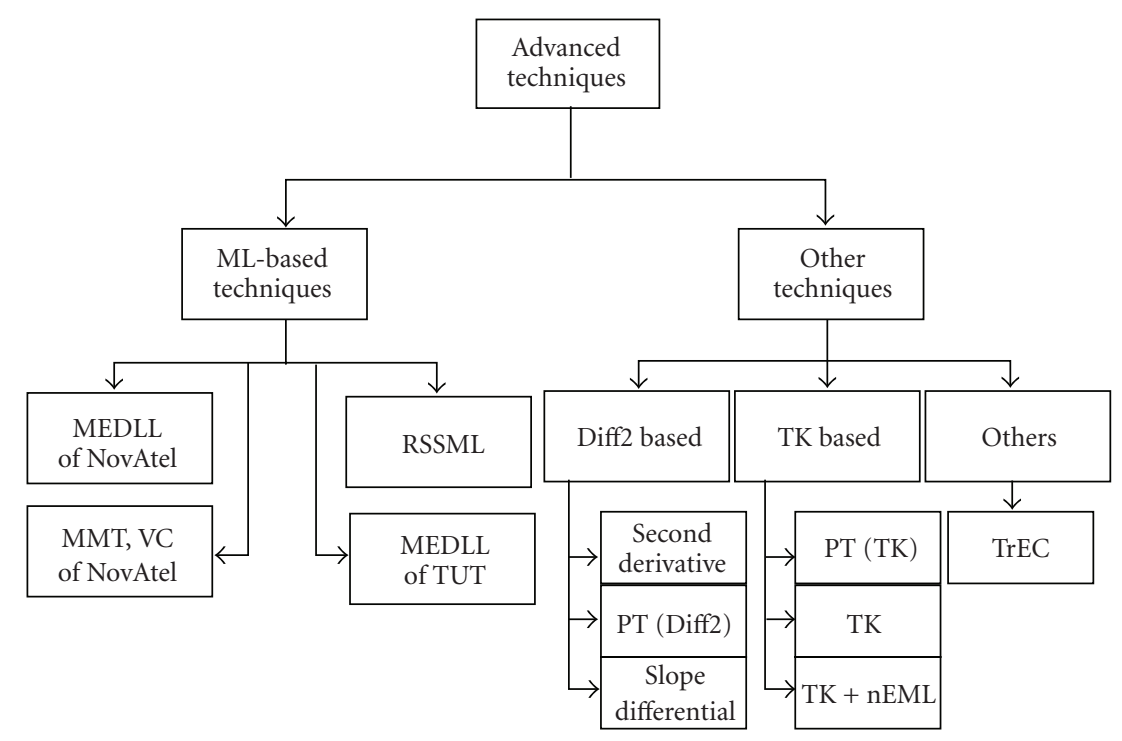

FIGURE 3: Classification of advanced techniques based on mitigation strategies.

TK-based technique is chosen in the context of the paper since it has been proved that it can give very good results in the delay-estimation process when used with CDMA type of signals, as presented in $[27,39]$. Most recently, TK has been studied also in closed-loop model for $\operatorname{SinBOC}(1,1)$, modulated two paths channel model, and its performance was one of the best among the considered algorithms [40]. One major limitation of TK-based technique is the fact that they are quite sensitive to the filtering stages (i.e., when infinite bandwidth is unavailable). The impact of the bandwidth limitation on TK performance is seldomly addressed in the literature, and hence, it is included in our algorithms' list for performance analysis under bandwidth limitation.

4.2. Combined Approach: Teager Kaiser and Narrow EML. A combined simplified approach with Teager Kaiser and narrow EML is implemented in order to justify the feasibility of having an nEML discrimination after the TK operation on the noncoherent correlation function. In this combined approach, TK operator is first applied to the noncoherent correlation function, and then nEML discrimination is applied to the TK output. The motivation for this combined approach comes from the fact that, when we apply TK operation to the noncoherent correlation function, it usually makes the main lobe of the noncoherent correlation function (after TK operation) much more steeper. This eventually reduces the effect of multipath in case of TK-based nEML (TK $+\mathrm{nEML})$ as compared to $\mathrm{nEML}$, as illustrated in Figure 4. In Figure 4, TK + nEML has a zero crossing at 0.014 chips away from the true delay whereas nEML has a zero crossing at 0.029 chips away from the true delay. Therefore, $\mathrm{TK}+\mathrm{nEML}$ has superior multipath performance (around 4.1 meters of multipath error) as compared to nEML (around 8.5 meters of multipath error) for this particular scenario. On the contrary, TK+nEML restricts the code delay search range (i.e., the range where we expect our true code delay to be located) to be much narrower

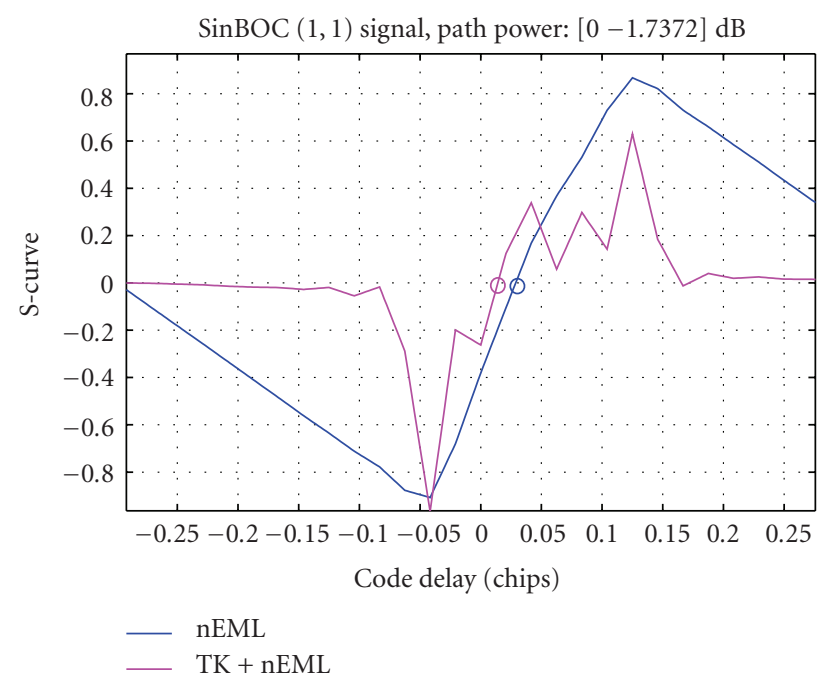

FIgure 4: S-curve in $\operatorname{SinBOC}(1,1)$ - modulated 2 path Rayleigh channel model, path delay: [0 0.0833] chips, path power: [0-1.7372] dB, C/N $: 100 \mathrm{~dB}-\mathrm{Hz}, \mathrm{BW}: 24 \mathrm{MHz}$.

as compared to nEML, which eventually increases the risk for the combined approach to lock at any of the false zero crossings, in cases when the initial coarse delays are poorly estimated.

4.3. Peak Tracking. Peak Tracking- (PT-) based techniques, namely, PT based on 2nd-order differentiation (PT(Diff2)) and PT based on Teager Kaiser (PT(TK)), are first proposed in $[27,41]$. Both of these techniques utilize the adaptive threshold computed from the estimated noise variance of the channel in order to decide on the correct code delay. These advanced techniques first generate competitive peaks which are above the computed adaptive threshold, 
as explained in Sections 5.1 and 5.2. For each of the competitive peak, a decision variable is formed based on the peak power, the peak position and the delay difference of the peak from the previous delay estimate. Finally, the PT techniques select the peak which has the maximum weight as being the best LOS candidate. PT(Diff2) is included in our list of multipath mitigation techniques for performance analysis to be presented in Sections 6 and 7, since it has superior multipath mitigation performance over PT(TK) [27].

\subsection{Reduced Search Space Maximum Likelihood Delay}

Estimator. In the presence of multipath, we recall that the received signal at the input of a GNSS receiver can be expressed as in (4). We rewrite (4) below for further clarification

$$
r(t)=\sum_{l=1}^{L} \alpha_{l} x\left(t-\tau_{l}\right) e^{j\left(2 \pi f_{D} t+\theta_{l}\right)}+\eta(t)
$$

In the above equation, as explained earlier, $x(t)$ is the spreadspectrum code, $\eta(t)$ is the white Gaussian noise, and $\alpha_{l}$, $\tau_{l}, \theta_{l}$ are the amplitude, delay, and phase of the $l$ th signal, respectively. For any GNSS signal, one of the most important parameter of interest is the LOS code delay. A conventional DLL (e.g., nEML) is not able to follow the LOS code delay accurately, since it does not take into consideration the bias contributed by the multipath components. The proposed RSSML attempts to compensate the multipath error contribution by estimating the multipath parameters along with the LOS signal. If $r(t)$ is observed for a certain time $T_{\text {coh }}$, that is short enough to assume that the parameters are constant, then the Maximum Likelihood Estimation (MLE) theory can be applied to estimate those parameters. The MLE principle states that the estimate of a certain parameter with the smallest mean square error is the estimate that maximizes the conditional probability density function of $r(t)$. According to MLE, RSSML calculates the estimated signal parameters (i.e., path delays, path amplitudes, and path phases), which minimize the mean square error of $L(\hat{\tau}, \hat{\alpha}, \hat{\theta})$, as specified in

$$
\begin{gathered}
L(\hat{\tau}, \hat{a}, \hat{\theta})=\int_{t-T_{\text {coh }}}^{t}[r(t)-s(t)]^{2} d t, \\
s(t)=\sum_{l=1}^{L} \hat{\alpha}_{l} x\left(t-\hat{\tau}_{l}\right) e^{j\left(2 \pi \hat{f}_{D} t+\hat{\theta}_{l}\right)} .
\end{gathered}
$$

Here, $s(t)$ is the estimate of the LOS as well as multipath signals, and we assume that the Doppler shift $f_{D}$ is correctly estimated by the carrier tracking loop (i.e., $\hat{f}_{D}=f_{D}$ ) and that all the multipath components experience similar Doppler shift (i.e., $f_{D_{l}}=f_{D}$ ). The first assumption is valid as long as we are at the fine tracking stage (i.e., signal has been tracked for a while). Equation (13) can be solved by setting the partial derivatives of $L(\hat{\tau}, \hat{\alpha}, \hat{\theta})$ to zero. The resulting equations for the lth signal can be written as follows in accordance with [25]:

$$
\begin{aligned}
& \hat{\tau}_{l}=\max _{\tau}\left[\operatorname{Re}\left\{\left[\begin{array}{c}
\mathcal{R}_{r x}(\tau)-\sum_{n=1}^{L} \hat{\alpha}_{n} \mathcal{R}_{\text {ideal }}\left(\tau-\hat{\tau}_{n}\right) e^{j \hat{\theta}_{n}} \\
n \neq l
\end{array}\right] e^{-j \hat{\theta}_{l}}\right\}\right], \\
& \left.\hat{\alpha}_{l}=\operatorname{Re}\left\{\left[\begin{array}{c}
\mathcal{R}_{r x}\left(\hat{\tau}_{i}\right)-\sum_{n=1}^{L} \hat{\alpha}_{n} \mathcal{R}_{\text {ideal }}\left(\hat{\tau}_{l}-\hat{\tau}_{n}\right) e^{j \hat{\theta}_{n}} \\
n \neq l
\end{array}\right] e^{-j \hat{\theta}_{l}}\right\}\right], \\
& \hat{\theta}_{l}=\arg \left[\begin{array}{c}
\mathcal{R}_{r x}\left(\hat{\tau}_{i}\right)-\sum_{n=1}^{L} \hat{\alpha}_{n} \mathcal{R}_{\text {ideal }}\left(\hat{\tau}_{l}-\hat{\tau}_{n}\right) e^{j \hat{\theta}_{n}} \\
n \neq l
\end{array}\right] .
\end{aligned}
$$

In the above equations, $\mathcal{R}_{r x}(\tau)$ is the received downconverted correlation function, and $\mathcal{R}_{\text {ideal }}(\tau)$ is the ideal reference correlation function, the expression of which can be found in [36]. Generally speaking, RSSML performs a nonlinear curve fit on the input correlation function which finds a perfect match from a set of ideal reference correlation functions with certain amplitude(s), phase(s), and delay(s) of the multipath signal. Conceptually, a conventional spreadspectrum receiver does the same thing, but for only one signal (i.e., the LOS signal). With the presence of multipath signal, RSSML tries to separate the LOS component from the combined signal by estimating all the signal parameters in MLE sense, which consequently achieves the best curve fit on the received input correlation function. The total number of path components $L$ is generally unknown to the receiver and, therefore, has to be estimated. One possible way to estimate $L$ is to compute the mean square error for $L=1,2, \ldots, L_{\max }$ number of paths and select $L$ with which we obtain the minimum mean square error. In this implementation, $L_{\max }$ is chosen such that the total number of path components does not exceed 3 (i.e., $L_{\max }=3$ ).

In a multicorrelator-based structure, the estimated LOS delay, theoretically, can be anywhere within the code delay window range of $\pm \tau_{W}$ chips, though in practice, it is quite likely to have a delay error around the previous delay estimate. The code delay window range essentially depends on the number of correlators (i.e., $M$ ) and the spacing between the correlators (i.e., $\Delta$ ) according to

$$
\tau_{W}= \pm \frac{(M-1)}{2} \Delta .
$$

For example, if 193 correlators are used with a correlator spacing of 0.0208 chips, then the resulting code-delay window range will be \pm 2 chips with respect to prompt correlator. 
Therefore, the LOS delay estimate can be anywhere within this \pm 2 chips window range. The ideal noncoherent reference correlation functions are generated for up to $L_{\max }$ paths only for the middle delay index (i.e., $((M+1) / 2)$ th delay index; for $M=193$, the middle delay index is 97). These ideal correlation functions for the middle delay index are generated offline and saved in a lookup table in memory. In real time, RSSML reads the correlation values from the lookup table, translates the ideal reference correlation functions at the middle delay index to the corresponding candidate delay index within the code delay window, and then computes the Minimum Mean Square Error (MMSE) for that specific delay candidate. Instead of considering all possible LOS delays within a predefined code delay window as delay candidates, the search space is first reduced to some competitive peaks which are generated based on the computed noise thresholds as explained in Section 5. This will eventually reduce the processing time required to compute the MMSE (i.e., MMSE needs to be computed only for the reduced search space).

\section{Implementation Issues for RSSML}

The implementation of RSSML is discussed here for better clarification. Setting the partial derivatives of (13) to zero yields a set of nonlinear equations, as presented in (14). To overcome the difficulty of solving these equations, the RSSML generates a set of ideal noncoherent reference correlation functions for the middle correlator of a certain code-delay window range with various multipath delays, phases, and amplitudes. This means that, we generate $s(t)$ in (13), by varying all multipath components for the middle correlator (e.g., the 97th correlator for a code-delay window range of \pm 2 chips with 0.0208 chips correlator spacing) of the code-delay window in order to obtain a discrete set of ideal noncoherent reference correlation functions. The set of multipath parameters can be specified as follows:

$$
\begin{aligned}
& A=\forall a\{0 \leq a \leq 1\}, \\
& T=\forall \tau\{0 \leq \tau \leq 1\}, \\
& \Theta=\forall \theta\{0 \leq \theta \leq 2 \pi\},
\end{aligned}
$$

where $|A|=p,|T|=q$, and $|\Theta|=r$ are the cardinalities of the sets $A, T$, and $\Theta$, respectively. The cardinality of each set will depend on the resolution of the multipath parameters within the given range. However, the complexity will increase as the cardinality of any set increases. The step-by-step procedure for RSSML is summarized below.

5.1. Step 1: Noise Estimation. The correlation values for early time delays (i.e., $<-1$ chip from the prompt correlator) are not affected by any multipath components since the multipath components are always delayed with respect to the LOS component. The noise level is estimated by taking the mean out-of-1-chip values at the early side from the prompt correlator of the normalized noncoherent correlation function as explained in Figure 5.

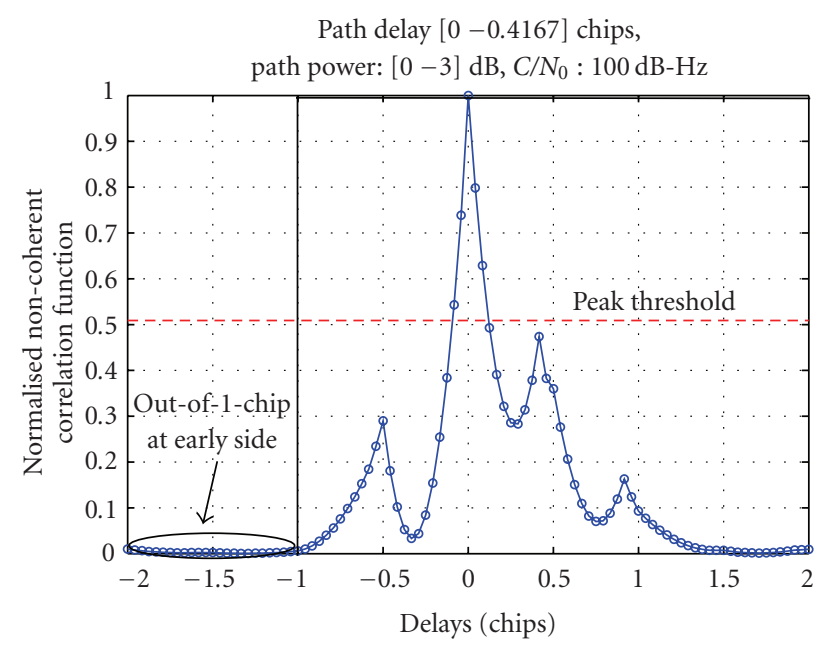

Figure 5: Noise estimation in $\operatorname{SinBOC}(1,1)$ - modulated 2 path fading channel model, path delay: [0-0.4167] chips, path power: [0-3] dB, C/N $: 100 \mathrm{~dB}-\mathrm{Hz}$.

5.2. Step 2: Competitive Peak Generation. The competitive peaks are those peaks which are generated based on the estimated noise level as obtained from step 1. A peak threshold is computed based on the estimated noise threshold plus some weighting factor as defined in [27]. The weighting factors are chosen in such a way that they reduce the possible risk that may arise due to the side lobes of the $\operatorname{SinBOC}(1,1)$ or $\mathrm{CBOC}(-)$ correlation. Therefore, the weighting factors chosen for $\operatorname{SinBOC}(1,1)$ and $\operatorname{CBOC}(-)$ modulations are slightly different from that of BPSK. It is worth to mention here that we use the same weighting factors for $\mathrm{CBOC}(-)$ and SinBOC $(1,1)$ modulations, since they have almost similar correlation shape. As shown in Figure 5, in this example case, there is only one competitive peak which is above the computed peak threshold. The search space is then reduced from a large number of correlators to some competitive delay candidates (serving here as competitive peaks).

\subsection{Step 3: Reference Correlation Functions Reading and} Translation. The RSSML first reads the ideal noncoherent reference correlation functions (which are generated offline for the middle/prompt correlator) from the lookup table. Next, it translates the correlation functions at the middle delay index to the corresponding candidate delay index within the code delay window for each competitive peak (which are already obtained from step 2). While doing the translation, RSSML truncates the ideal reference correlation values to zero which fall outside the code delay window range (i.e., correlation values outside \pm 2 chips from the middle correlator are truncated to zero).

5.4. Step 4: MMSE Computation. The RSSML computes the MMSE for each candidate delay index corresponding to a competitive peak obtained from step 2 .

5.5. Step 5: LOS Delay Estimation. The candidate delay index with the lowest MMSE is chosen as the estimated LOS delay. 


\section{Semianalytical Running Average Error}

The most typical way to evaluate the performance of a multipath mitigation technique is via Multipath Error Envelopes (MEE). Typically, two paths, either in phase or out of phase, are assumed to be present, and the multipath errors are computed for multipath delays up to 1.2 chips at maximum, since the multipath errors become less significant after that. The upper multipath error envelope can be obtained when the paths are in phase and the lower multipath error envelope when the paths are out of phase (i.e., $180^{\circ}$ phase difference). In MEE analysis, several simplifying assumptions are usually made in order to distinguish the performance degradation caused by the multipath errors only. Such assumptions include zero Additive-White-Gaussian-Noise (AWGN), ideal infinite-length PRN codes, and zero residual Doppler. Under these assumptions, the correlation $\mathcal{R}_{\mathrm{rx}}(\tau)$ between the reference code of modulation type MOD (e.g., BPSK or CBOC(-)) and the received MOD-modulated signal via an $L$-path channel can be written as

$$
\mathcal{R}_{\mathrm{rX}}(\tau)=\sum_{l=1}^{L} \alpha_{l} e^{j \theta_{l}} \mathcal{R}_{\mathrm{MOD}}\left(\tau-\tau_{l}\right),
$$

where $\alpha_{l}, \theta_{l}, \tau_{l}$ are the amplitude, phase, and delay, respectively, of the $l$ th path; and $R_{\mathrm{MOD}}(\tau)$ is the autocorrelation function of a signal with modulation type MOD. The analytical expressions for MEEs become complicated in the presence of more than two paths due to the complexity of channel interactions. Therefore, an alternative MonteCarlo simulations-based approach is proposed herein for multipath error analysis in more than one path scenarios (i.e, for $L \geq 2$ ). First, a sufficient number of random realizations, $N_{\text {random }}$ are generated (i.e., in the simulations, we choose $N_{\text {random }}$ equals to 2000), and then we look at absolute mean error for each path delay over $N_{\text {random }}$ points. The objective here is to analyze the multipath performance of various tracking techniques in the presence of more than two channel paths, which may occur in urban or indoor scenarios.

The following assumptions are made while running the simulations for generating the curves of Running Average Error (RAE). The channel follows a decaying Power Delay Profile (PDP), which can be expressed by the equation:

$$
\alpha_{l}=\alpha_{l} \exp ^{-\mu\left(\tau_{l}-\tau_{1}\right)},
$$

where $\left(\tau_{l}-\tau_{1}\right) \neq 0$ for $l>1, \mu$ is the PDP coefficient (assumed to be uniformly distributed in the interval $[0.05 ; 0.1]$, when the path delays are expressed in samples). The channel path phases $\theta_{l}$ are uniformly distributed in the interval $[0 ; 2 \pi]$, and the number of channel paths $L$ is uniformly distributed between 2 and $L_{\max }$, where $L_{\max }$ is set to 4 in the simulations. A constant successive path spacing $x_{\mathrm{ct}}$ is chosen in the range $[0 ; 1.167]$ chips with a step of 0.0417 chips (which will define the multipath delay axis in the running average error curves). It is worth to mention here that the number of paths reduced to one LOS path when $x_{\mathrm{ct}}=0$. The successive path delays can be found using the formula $\tau_{l}=l x_{\mathrm{ct}}$ in chips. Therefore, for each channel realization (which is a combination of amplitudes $\vec{\alpha}=\alpha_{1}, \ldots, \alpha_{L}$, phases $\vec{\theta}=\theta_{1}, \ldots, \theta_{L}$, fixed path spacings, and the number of channel paths $L$ ), a certain LOS delay is estimated $\hat{\tau}_{1}(\vec{\alpha}, \vec{\theta}, L)$ from the zero crossing of the discriminator function (i.e., $D(\tau)=0$ ), when searched in the linear range of $D(\tau)$. The estimation error due to multipath is $\hat{\tau}_{1}(\vec{\alpha}, \vec{\theta}, L)-\tau_{1}$, where $\tau_{1}$ is the true LOS path delay. The RAE curves are generated in accordance with [42]. RAE is actually computed from the area enclosed within the multipath error and averaged over the range of the multipath delays from zero to the plotted delay values. Therefore, in order to generate the RAE curves, the absolute mean error is computed for all $N_{\text {random }}$ random points via

$$
\operatorname{AME}\left(x_{c t}\right)=\operatorname{mean}\left(\left|\hat{\tau}_{1}(\vec{\alpha}, \vec{\theta}, L)-\tau_{1}\right|\right),
$$

where $\operatorname{AME}\left(x_{c t}\right)$ is the mean of absolute multipath error for the successive path delay $x_{c t}$. Now, the running average error for each particular delay in the range $[0 ; 1.167]$ chips can be computed as follows:

$$
\operatorname{RAE}\left(x_{\mathrm{ct}}\right)=\frac{\sum_{i=1}^{i} \operatorname{AME}\left(x_{\mathrm{ct}}\right)}{i},
$$

where $i$ is the successive path delay index and $\operatorname{RAE}\left(x_{\mathrm{ct}}\right)$ is the RAE for the successive path delay $x_{\mathrm{ct}}$. The RAE curves for three different modulations are shown in Figure 6.

\section{Simulation Results}

The semianalytical results from Section 6 have also been validated via simulations in fading multipath channels. Simulations have been carried out in closely spaced multipath scenarios for BPSK-, $\operatorname{SinBOC}(1,1)-$, and CBOC(-)modulated signals for a finite front-end bandwidth. The simulation profile is summarized in Table 1. Rayleigh fading channel model is used in the simulation, where the number of channel paths follows a uniform distribution between two and four. The successive path separation is random between 0.02 and 0.35 chips. The channel paths are assumed to obey a decaying PDP following (18), where $\mu=0.1$ (when the path delays are expressed in samples). The received signal was sampled at $N_{s}=48,24$, and 4 for BPSK-, $\operatorname{SinBOC}(1,1)$ - and CBOC(-)- modulated signals, respectively. $N_{s}$ varies in order to have the same number of samples per chip for all the three cases.

The received signal duration is 800 milliseconds (ms) or 0.8 seconds for each particular $C / N_{0}$ level. The tracking errors are computed after each $N_{c} N_{\mathrm{nc}}$ ms (in this case, $N_{c} N_{\text {nc }}=20 \mathrm{~ms}$ ) interval. In the final statistics, the first $600 \mathrm{~ms}$ are ignored in order to remove the initial error bias that may come from the delay difference between the received signal and the locally generated reference code. Therefore, for the above configuration (i.e., code loop filter parameters and the first path delay of 0.2 chips), the leftover tracking errors after $600 \mathrm{~ms}$ are mostly due to the effect of multipath only, as shown in Figure 7. We run the simulations for 100 random realizations, which give a total of $10 * 100$ $=1000$ statistical points, for each $C / N_{0}$ level. The RootMean-Square-Errors (RMSE) of delay estimates are plotted 


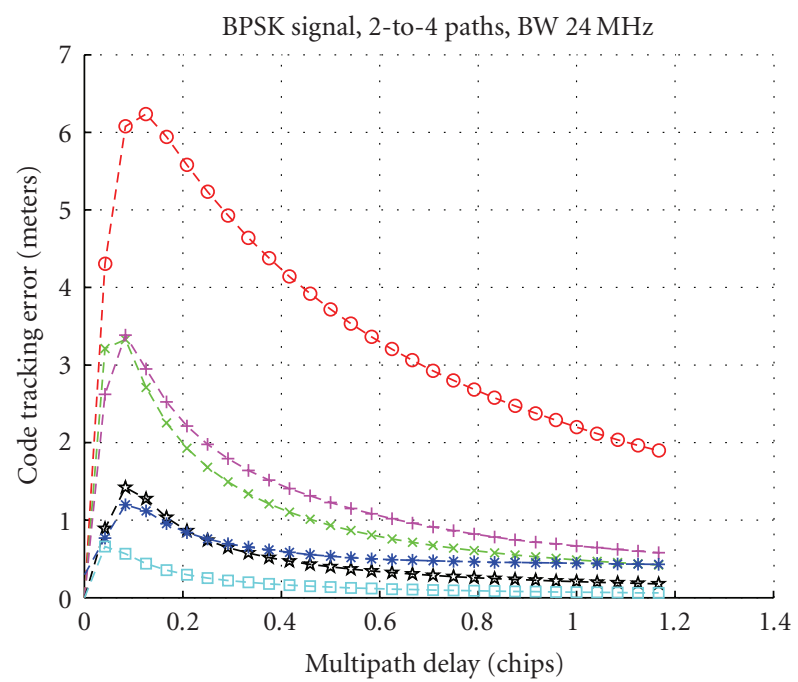

(a) BPSK signal

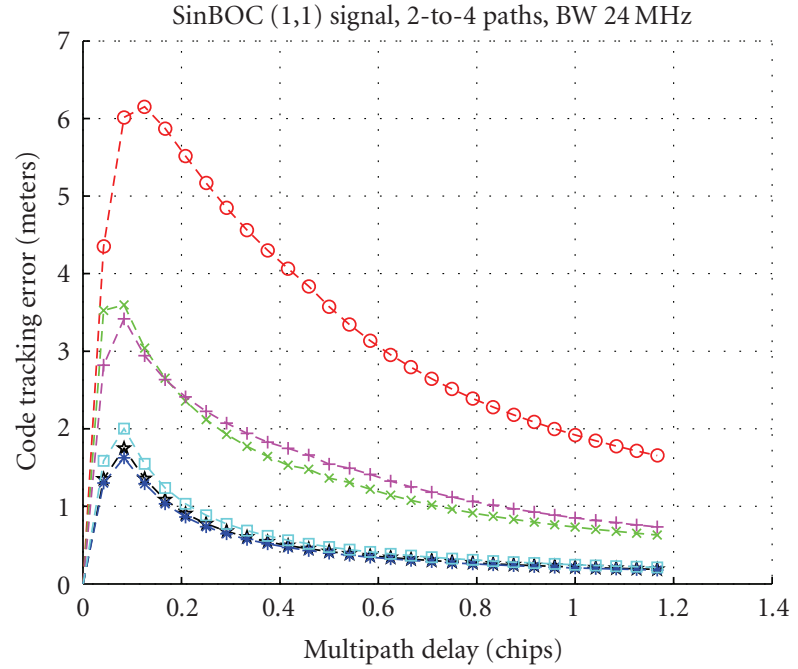

(b) $\operatorname{Sin} B O C(1,1)$ signal

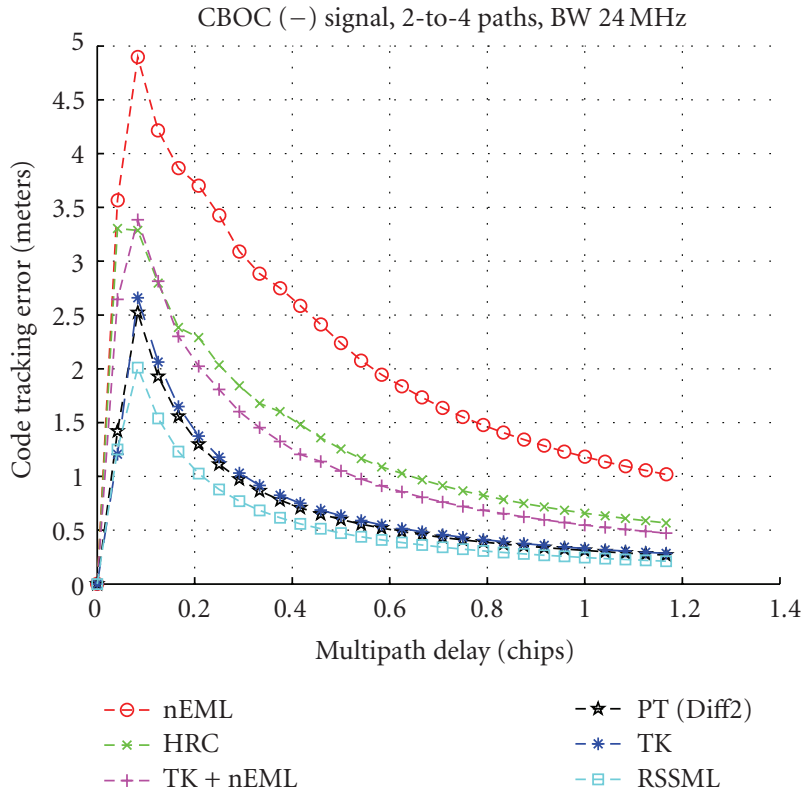

(c) $\mathrm{CBOC}(-)$ signal

FIgURE 6: RAE for BPSK, $\operatorname{SinBOC}(1,1)$, and CBOC(-) signals.

in meters, by using the relationship $\mathrm{RMSE}_{m}=\mathrm{RMSE}_{\mathrm{chips}} c T_{c}$, where $c$ is the speed of light, $T_{c}$ is the chip duration, and $\mathrm{RMSE}_{\text {chips }}$ is the RMSE in chips. RMSE versus $C / N_{0}$ plots for the given multipath-channel profile are shown in Figure 8. Additionally, a RMSE versus $C / N_{0}$ plot is presented in Figure 9 for $\operatorname{SinBOC}(1,1)$ - modulated single path signal in order to show the performance of the mitigation techniques in the absence of any multipath. In this nomultipath scenario, nEML has the best tracking performance from $C / N_{0} 35 \mathrm{~dB}-\mathrm{Hz}$ and higher whereas RSSML showed the best tracking performance in $30 \mathrm{~dB}-\mathrm{Hz}$, and slightly worse performance than nEML from $C / N_{0} 35 \mathrm{~dB}-\mathrm{Hz}$ and higher.

\section{Performance Comparison}

Table 2 shows the comparison between the different discussed techniques in terms of closely spaced multipath performance, semianalytical running average error performance, correlator requirement (in other words, code delay window length at the tracking stage), a priori information needed as input, channel estimation requirement, memory requirement, and complexity analysis as a whole. This comparison is solely based on the simulation results described in Sections 6 and 7.

It can be seen from Figure 8 that the proposed RSSML showed the best multipath performance in closely spaced 


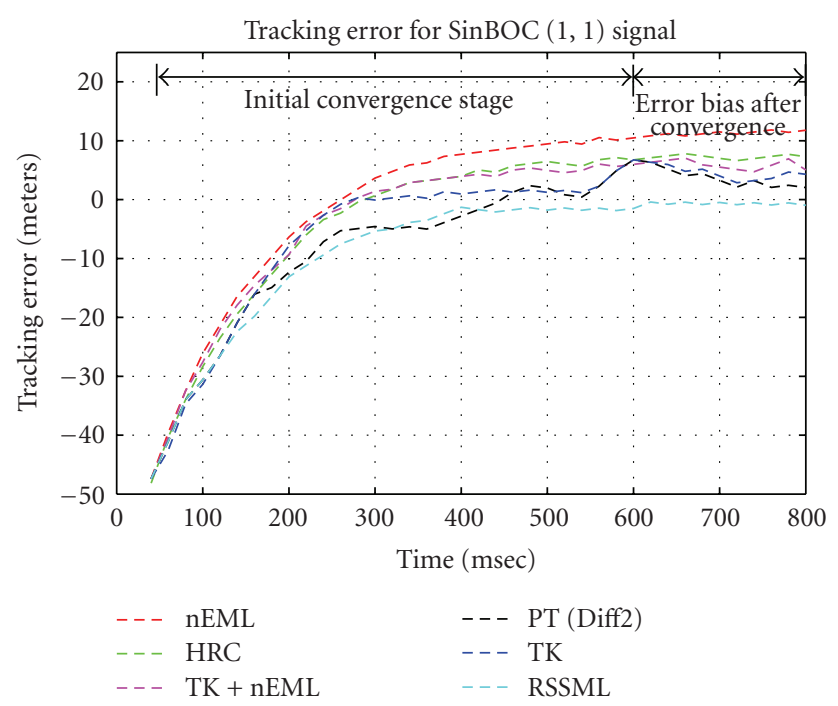

FIGURE 7: One snapshot of delay tracking for $\operatorname{SinBOC}(1,1)$ signal in 3 path fading channel with $24 \mathrm{MHz}$ BW.

TABLE 1: Simulation profile description.

\begin{tabular}{ll}
\hline Parameter & Value \\
\hline Channel model & Rayleigh fading channel \\
Number of paths & (between 2 to 4) \\
Path power & $\begin{array}{l}\text { Decaying PDP with } \mu=0.1 \\
\text { Random between } 0.02 \text { and }\end{array}$ \\
Path spacing & 0.35 chips \\
Path phase & Random between 0 and $2 \pi$ \\
Oversampling factor, $N_{s}$ & {$[48,24,4]$} \\
E-L Spacing, $\Delta_{\text {EL }}$ & 0.0833 chips \\
Number of Correlators, $M$ & 193 \\
Double-sided Bandwidth, BW & $24 \mathrm{MHz}$ \\
Filter type & FIR \\
Filter order & 6 \\
Coherent integration, $N_{c}$ & $20 \mathrm{~ms}$ \\
Noncoherent integration, $N_{\mathrm{nc}}$ & $1 \mathrm{block}$ \\
Initial delay error & $\pm 0.1 \mathrm{chips}$ \\
First path delay & $0.2 \mathrm{chips}$ \\
Code tracking loop bandwidth & $2 \mathrm{~Hz}$ \\
Code tracking loop order & $1 \mathrm{st}$ order \\
\hline
\end{tabular}

two to four paths fading channel model for all three modulation types. All other techniques have varying multipath performance with varying $C / N_{0}$ and varying modulation types. In general, PT(Diff2) performs better for $\operatorname{SinBOC}(1,1)$ and $\mathrm{CBOC}(-)$ signals whereas HRC performs better for all three modulations, but only in good $\mathrm{C} / \mathrm{N}_{0}$ (i.e., $40 \mathrm{~dB}$ $\mathrm{Hz}$ and higher). It is interesting to note here that all the techniques except the proposed RSSML tend to show similar performance (within few meters of error bounds) in this two to four paths fading channel profile with a reasonably high PDP factor 0.1, as seen in Figure 8.

The semianalytical RAE performance is shown in Figure 6. It is obvious from Figure 6 that the proposed RSSML showed superior performance in terms of RAE as compared to other techniques in this no noise two to four paths static channel model. Among other techniques, PT(Diff2) and TK showed very good performance followed by HRC and TK + nEML. The RAE analysis is quite theoretical from two perspectives: firstly, the delay estimation is a one-shot estimate and does not really include any tracking loop in the process, and secondly, the analysis is usually carried out with ideal noise free assumption. These facts probably explain the reason why an algorithm which performs very good with respect to RAE may not necessarily provide the same performance in more realistic closed-loop fading channel model, especially in the presence of more than two channel paths. However, MEE or RAE analysis has been widely used by the research community as an important tool for analyzing the multipath performance due to simpler implementation and also due to the fact that it is hard to isolate multipath from other GNSS error sources in real life.

The complexity of any multipath mitigation technique mainly depends on the correlation structure and the implementation issues concerning channel estimation, correlator requirement, required number of mathematical operations, memory requirement, and so on. The advanced mitigation techniques are usually complex, since they generally utilize a large number of correlators for channel estimation, which are then used to estimate the first arriving path delay. Among the advanced techniques, the proposed RSSML is the most complex one, since it requires a large set of reference correlation functions which are generated offline to be used as a-priori information while estimating the code delay of first arriving path (please visit Section 5 for details). The memory size will eventually depend on few factors including the maximum number of paths to be considered, the correlator spacing, the number of correlators and the resolution of each multipath parameter (i.e., path delays, path phases, and path amplitudes). In the current MATLAB implementation, the RSSML requires approximately 14 megabytes of memory for each particular modulation with maximum number of paths set to 3 , the correlator spacing set to 0.0208 chips, the number of correlators for window length of 4 chips set to 193. However, it is possible to reduce the memory requirement by adjusting the parameters appropriately. The impact of memory optimization is not analyzed here, and hence, it is kept open for future research.

\section{Conclusions}

Multipath is one of the major dominant sources in highprecision-oriented GNSS applications. Many receiver architectures exist in the market which employ a variety of 


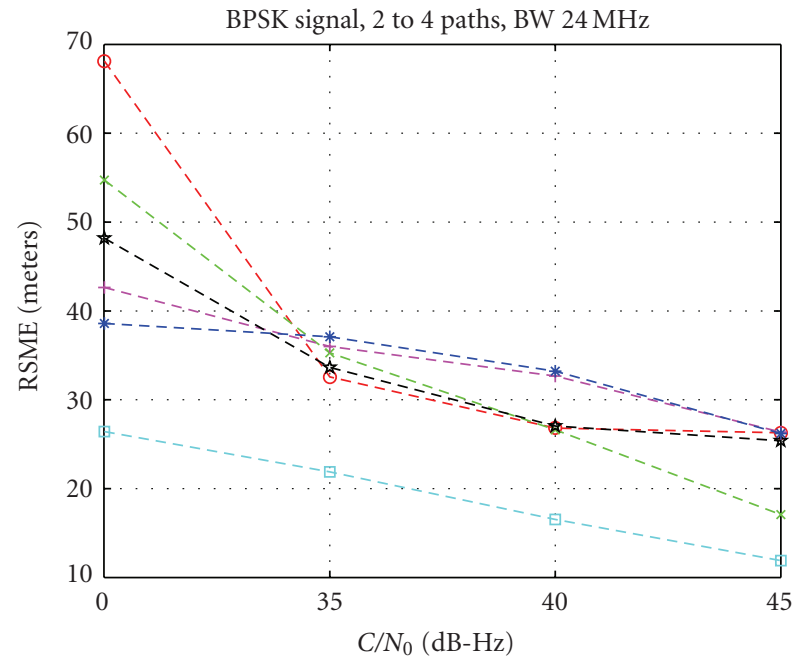

(a) BPSK signal

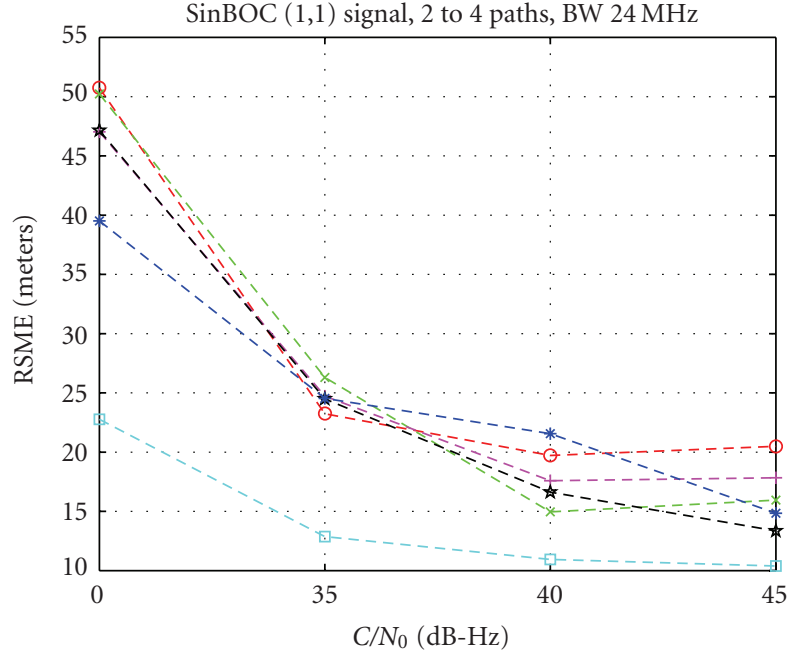

(b) $\operatorname{Sin} B O C(1,1)$ signal

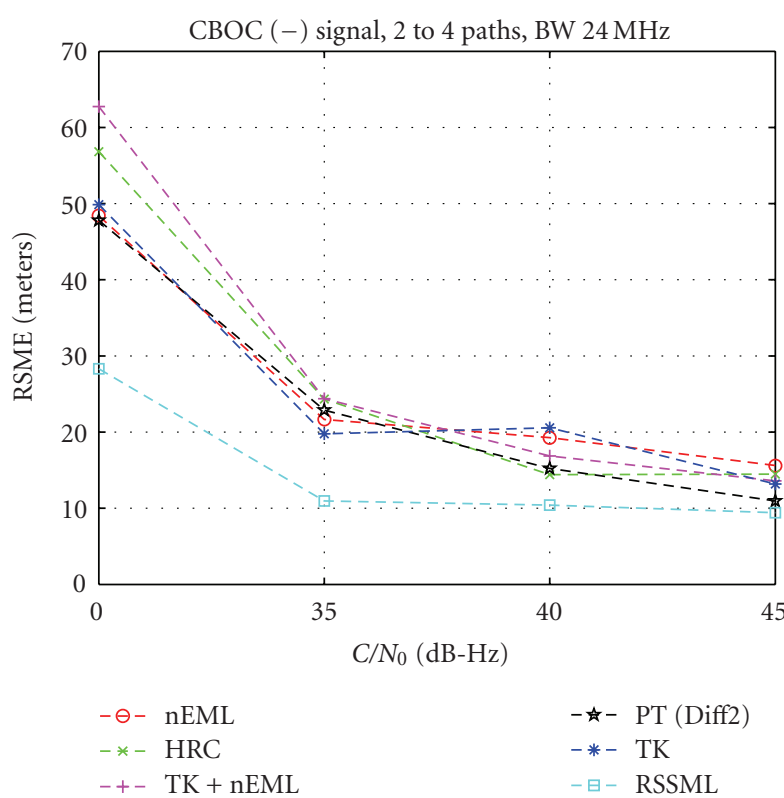

(c) $\mathrm{CBOC}(-)$ signal

Figure 8: RMSE versus $C / N_{0}$ plots for 2 to 4 paths Rayleigh fading channel in $24 \mathrm{MHz} B W$.

multipath mitigation techniques. Most of these techniques provide very good multipath mitigation for medium-to-long delay multipath. However, the multipath studies presented in most of the research papers are based on only two paths assumption, which is rather optimistic. In this study, a novel Reduced Search Space Maximum Likelihood delay estimator was proposed and the multipath performance was studied for short delay multipath where the number of paths varied between two and four. The multipath performance of the newly proposed technique along with the state-ofthe-art DLLs, and other advanced techniques were presented via running average error sense and also via root-meansquare-error sense. Three different modulation types were considered including the newly proposed CBOC modulation (chosen as the modulation technique for Galileo E1 signal).
It was shown that the RSSML, in general, achieved the best multipath mitigation performance for all three different signals in this two-to-four paths closely spaced multipath profile. Simulation results show that the proposed RSSML offers a viable solution by increasing the position accuracy in the presence of closely spaced multipath, especially in dense urban areas where the number of significant paths can be higher than two. On the contrary, the proposed method increases the receiver complexity, since it is based on multicorrelator-based structure, and at the same time, it requires a good amount of memory to keep the reference noncoherent correlation functions available for computing the MMSE. Therefore, RSSML and other advanced multipath mitigation techniques presented here are more suitable for professional receivers due to their relatively high complexity 
TABLE 2: Comparative performance of multipath mitigation techniques.

\begin{tabular}{|c|c|c|c|c|c|c|}
\hline & nEML & HRC & $\mathrm{TK}+\mathrm{nEML}$ & PT(Diff2) & TK & RSSML \\
\hline $\begin{array}{l}\text { Closely spaced } \\
\text { multipath } \\
\text { performance } \\
\end{array}$ & Moderate & Good & Moderate & Good & Moderate & Best \\
\hline $\begin{array}{l}\text { Running average } \\
\text { error performance }\end{array}$ & Fair & Good & Good & Very Good & Very Good & Best \\
\hline $\begin{array}{l}\text { Correlator } \\
\text { requirement (No. of } \\
\text { Corr.) }\end{array}$ & Few (3) & Few (5) & Few (5) & Many $(100+)$ & Many $(100+)$ & Many $(100+)$ \\
\hline A priori information & $\begin{array}{l}\text { Coarse delay } \\
\text { estimate }\end{array}$ & $\begin{array}{l}\text { Coarse delay } \\
\text { estimate }\end{array}$ & $\begin{array}{l}\text { Coarse delay } \\
\text { estimate }\end{array}$ & $\begin{array}{l}\text { Coarse delay } \\
\text { estimate }\end{array}$ & $\begin{array}{l}\text { Coarse estimate } \\
\text { delay }\end{array}$ & $\begin{array}{l}\text { A large set of } \\
\text { reference } \\
\text { correlation } \\
\text { functions }\end{array}$ \\
\hline $\begin{array}{l}\text { Channel estimation } \\
\text { required? }\end{array}$ & No & No & No & $\begin{array}{l}\text { Yes (noise } \\
\text { computation) }\end{array}$ & $\begin{array}{l}\text { Yes (noise } \\
\text { computation) }\end{array}$ & $\begin{array}{l}\text { Yes (noise } \\
\text { computation) }\end{array}$ \\
\hline Memory requirement & None & None & None & None & None & High \\
\hline Complexity & Low & Low & Moderate & Fair & Fair & High \\
\hline
\end{tabular}

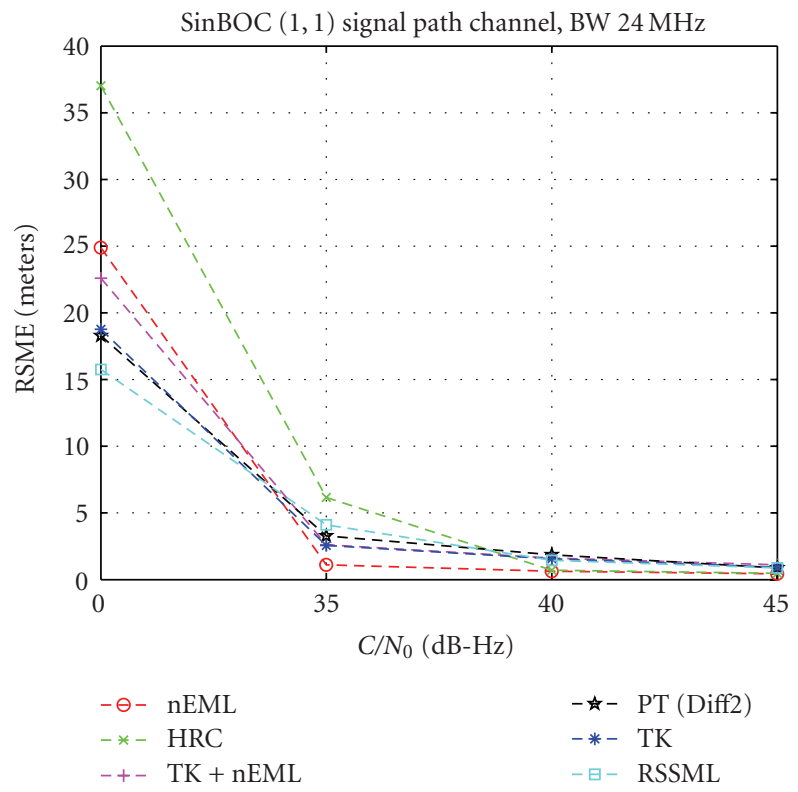

Figure 9: RMSE versus $C / N_{0}$ plot for single path $\operatorname{SinBOC}(1,1)$ signal in $24 \mathrm{MHz}$ BW.

whereas for mass-market receivers, nEML and HRC are still the best tradeoff between performance and complexity.

\section{Acknowledgments}

This work was carried out in the project "Future GNSS Applications and Techniques (FUGAT)" funded by the Finnish Funding Agency for Technology and Innovation (Tekes). This work has also been supported by the Academy of Finland, which is gratefully acknowledged. The authors would also like to thank Nokia Foundation and Tampere Doctoral Programme in Information Science and Engineering (TISE) for their financial support.

\section{References}

[1] A. J. V. Dierendonck, P. Fenton, and T. Ford, "Theory and performance of narrow correlator spacing in a GPS receiver," Journal of the Institute of Navigation, vol. 39, no. 3, pp. 265283, 1992.

[2] G. Fock, J. Baltersee, P. Schulz-Rittich, and H. Meyr, "Channel tracking for rake receivers in closely spaced multipath environments," IEEE Journal on Selected Areas in Communications, vol. 19, no. 12, pp. 2420-2431, 2001.

[3] P. Fine and W. Wilson, "Tracking algorithms for GPS offset carrier signals," in Proceedings of the ION National Technical Meeting, January 1999.

[4] J. Baltersee, G. Fock, and P. Schulz-Rittich, "Adaptive codetrackingreceiver for direct-sequence Code Division Multiple Access (CDMA)communications over multipath fading channels and method for signalprocessing in a RAKE receiver," US Patent Application Publication, US2001/0014114 A1 (Lucent Technologies), August 2001.

[5] R. Bischoff, R. Häb-Umbach, W. Schulz, and G. Heinrichs, "Employment of a multipath receiver structure in a combined GALILEO/UMTS receiver," in Proceedings of the 55th Vehicular Technology Conference (VTC '02), pp. 1844-1848, May 2002.

[6] K. Chen and L. D. Davisson, "Analysis of SCCL as a PN-code tracking loop," IEEE Transactions on Communications, vol. 42, no. 11, pp. 2942-2946, 1994.

[7] M. Laxton, -Analysis and simulation of a new code tracking loopfor GPS multipath mitigation, M.S. thesis, Air Force Institute of Technology, 1996.

[8] M. Irsigler and B. Eissfeller, "Comparison of multipath mitigation techniques with consideration of future signal structures," in Proceedings of the 16th International Technical Meeting of the Satellite Division of the Institute of Navigation (ION GNSS '03), pp. 2584-2592, Portland, Ore, USA, September 2003.

[9] G. A. McGraw and M. S. Braasch, "GNSS multipath mitigation using gated and high resolution correlator concepts," in Proceedings of the he National Technical Meeting of the Satellite Division of the Insitute of Navigation, San Diego, Calif, USA, January 1999. 
[10] J. W. Betz and K. R. Kolodziejski, "Extended theory of earlylate code tracking for a bandlimited GPS receiver," Navigation, Journal of the Institute of Navigation, vol. 47, no. 3, pp. $211-$ 226, 2000.

[11] M. S. Braasch, "Performance comparison of multipath mitigating receiver architectures," in Proceedings of the IEEE Aerospace Conference, pp. 31309-31315, Big Sky, Mont, USA, March 2001.

[12] H. Hurskainen, E. S. Lohan, X. Hu, J. Raasakka, and J. Nurmi, "Multiple gate delay tracking structures for GNSS signals and their evaluation with Simulink, SystemC, and VHDL," International Journal of Navigation and Observation, vol. 2008, Article ID 785695, 17 pages, 2008.

[13] Garin and Rousseau, "Enhanced strobe correlator multipath rejection for code \& carrier," in Proceedings of the 10th International Technical Meeting of the Satellite Division of the Institute of Navigation (ION GPS '97), vol. 1, pp. 559-568, Kansas City, Mo, USA, September 1997.

[14] J. Jones, P. Fenton, and B. Smith, "Theory and performance of the pulse aperture correlator," Tech. Rep., Novatel, Alberta, Canada, September 2004.

[15] L. R. Weill, "Multipath mitigation-how good can it get with new signals?" GPS World, vol. 16, no. 6, pp. 106-113, 2003.

[16] R. Fante, "Unambiguous tracker for GPS binary-offset carrier signals," in Proceedings of the National Technical Meeting of the Institute of Navigation (ION NTM '03), Albuquerque, NM, USA, 2003.

[17] R. Fante, "Unambiguous first-order tracking loop M-Code," MITRE Technical Report MTR 94B0000040, July 2004.

[18] P. A. Bello and R. L. Fante, "Code tracking performance for novel unambiguous M-Code time discriminators," in Proceedings of the National Technical Meeting of the Institute of Navigation (ION NTM '05), pp. 293-298, San Diego, Calif, USA, January 2005.

[19] M. Z. H. Bhuiyan, Analyzing code tracking algorithms for galileo open service signal, M.S. thesis, Tampere University of Technology, August 2006.

[20] A. J. V. Dierendonck and M. S. Braasch, "Evaluation of GNSS receiver correlation processing techniques for multipath and noise mitigation," in Proceedings of the National Technical Meeting of the Institute of Navigation (ION NTM '97), pp. 207215, Santa Monica, Calif, USA, January 1997.

[21] Townsend and Fenton, "Practical approach to the reduction of pseudorange multipath errors in a L1 GPS receiver," in Proceedings ofthe 7th International Technical Meeting of the Satellite Division of theInstitute of Navigation (ION-GPS '94), vol. 1, pp. 143-148, Salt Lake City, Utah, USA, September 1994.

[22] J. M. Sleewaegen and F. Boon, "Mitigating short-delay multipath: apromising new technique," in Proceedings of the International Technical Meeting of the Satellite Division of the Institute of Navigation (ION GPS '01), pp. 204-213, Salt Lake City, Utah,USA, September 2001.

[23] M. Z.H. Bhuiyan, E. S. Lohan, and M. Renfors, "A slopebased multipath estimation technique for mitigating shortdelay multipath in GNSS receivers," in Proceedings of the IEEE International Symposium on Circuits and Systems (ISCAS '10), pp. 3573-3576, 2010.

[24] R. D. J. V. Nee, “The multipath estimating delay lock loop," in Proceedings of the IEEE 2nd International Symposium on Spread Spectrum Techniques and Applications, pp. 39-42, Yokohama, Japan, november 1992.
[25] Richard D. J. van Nee, Siereveld, Patrick C. Fenton, and Bryan R. Townsend, "Multipath estimating delay lock loop: approaching theoretical accuracy limits," in Proceedings of the IEEE PLANS, Position Location and Navigation Symposium, vol. 1, pp. 246-251, 1994.

[26] B. Townsend, D. J. R. van Nee, P. Fenton, and K. Van Dierendonck, "Performance evaluation of the multipath estimating delay lock loop," in Proceedings of the National Technical Meeting, pp. 277-283, Anaheim, Calif, USA, January 1995.

[27] M. Z. H. Bhuiyan, E. S. Lohan, and M. Renfors, "Code tracking algorithms for mitigating multipath effects in fading channels for satellite-based positioning," EURASIP Journal on Advances in Signal Processing, vol. 2008, Article ID 863629, 2008.

[28] Lawrence R. Weill, "GPS multipath mitigation by means of correlator reference waveform design," in Proceedings of the National Technical Meeting, Institute of Navigation (NTM '97), pp. 197-206, Santa Monica, Calif, USA, January1997.

[29] C. Lee, S. Yoo, S. Yoon, and S. Y. Kim, "A novel multipath mitigation scheme based on slope differential of correlator output for Galileo systems," in Proceedings of the 8th International Conference Advanced Communication Technology (ICACT '06), vol. 2, pp. 1360-1363, February 2006.

[30] P. C. Fenton and J. Jones, "The theory and performance of NovAtel Inc.'s Vision Correlator," in Proceedings of the 18th International Technical Meeting of the Satellite Division of The Institute of Navigation (ION GNSS '05), pp. 2178-2186, Long Beach, Calif, USA, September 2005.

[31] L. Weill, "Multipath mitigation using modernized GPS signals: how good can it get?" in Proceedings of the International Technical Meeting of the Satellite Division of the Institute of Navigation (ION GPS '02), pp. 493-505, Palm Springs, Calif, USA, September 2002.

[32] E. S. Lohan, A. Lakhzouri, and M. Renfors, "Feedforward delay estimators in adverse multipath propagation for galileo and modernized GPS signals," EURASIP Journal on Applied Signal Processing, vol. 2006, Article ID 50971, 2006.

[33] M. Z. H Bhuiyan, E. S. Lohan, and M. Renfors, "A reduced search space maximum likelihood delay estimator for mitigating multipath effects in satellite-based positioning," in Proceedings of the 13th International Association of Institute of Navigation, October 2009.

[34] European Space Agency, "Galileo Open Service Signal In Space Interface Control Document," OS SIS ICD, Draft 1, February 2008.

[35] E. Kaplan and C. J. Hegarty, Understanding GPS: Principles and Applications, Artech House, Norwood, Mass, USA, 2nd edition, 2006.

[36] E. S. Lohan, A. Lakhzouri, and M. Renfors, "Binary-OffsetCarrier modulation techniques with applications in satellite navigation systems," Wireless Communications and Mobile Computing, vol. 7, no. 6, pp. 767-779, 2007.

[37] M. S. Braasch and A. J. Van Dierendonck, "GPS receiver architectures and measurements," Proceedings of the IEEE, vol. 87, no. 1, pp. 48-64, 1999.

[38] R. Hamila, E. S. Lohan, and M. Renfors, "Subchip multipath delay estimation for downlink WCDMA system based on Teager-Kaiser operator," IEEE Communications Letters, vol. 7, no. 1, pp. 1-3, 2003.

[39] E. S. Lohan, R. Hamila, A. Lakhzouri, and M. Renfors, "Highly efficient techniques for mitigating the effects of multipath propagation in DS-CDMA delay estimation," IEEE Transactions on Wireless Communications, vol. 4, no. 1, pp. 149-162, 2005. 
[40] M. Z. H. Bhuiyan, E. S. Lohan, and M. Renfors, "Multipath mitigation performance of multi-correlator based code tracking algorithms in closedand open loop model," in Proceedings of the The 15th European Wireless Conference, pp. 84-89, May 2009.

[41] M. Z. H. Bhuiyan, E. S. Lohan, and M. Renfors, "Peak tracking algorithm for Galileo-based positioning in multipath fading channels," in Proceedings of the IEEE International Conference on Communications (ICC '07), pp. 5927-5932, June 2007.

[42] G. W. Hein, J.-A. Avila-Rodriguez, S. Wallner et al., "MBOC: the new optimized spreading modulation recommended for GALILEO L1 OS and GPS L1C," in Proceedings of IEEE Position, Location and Navigation Symposium (ION PLANS '06), pp. 883-892, April 2006. 

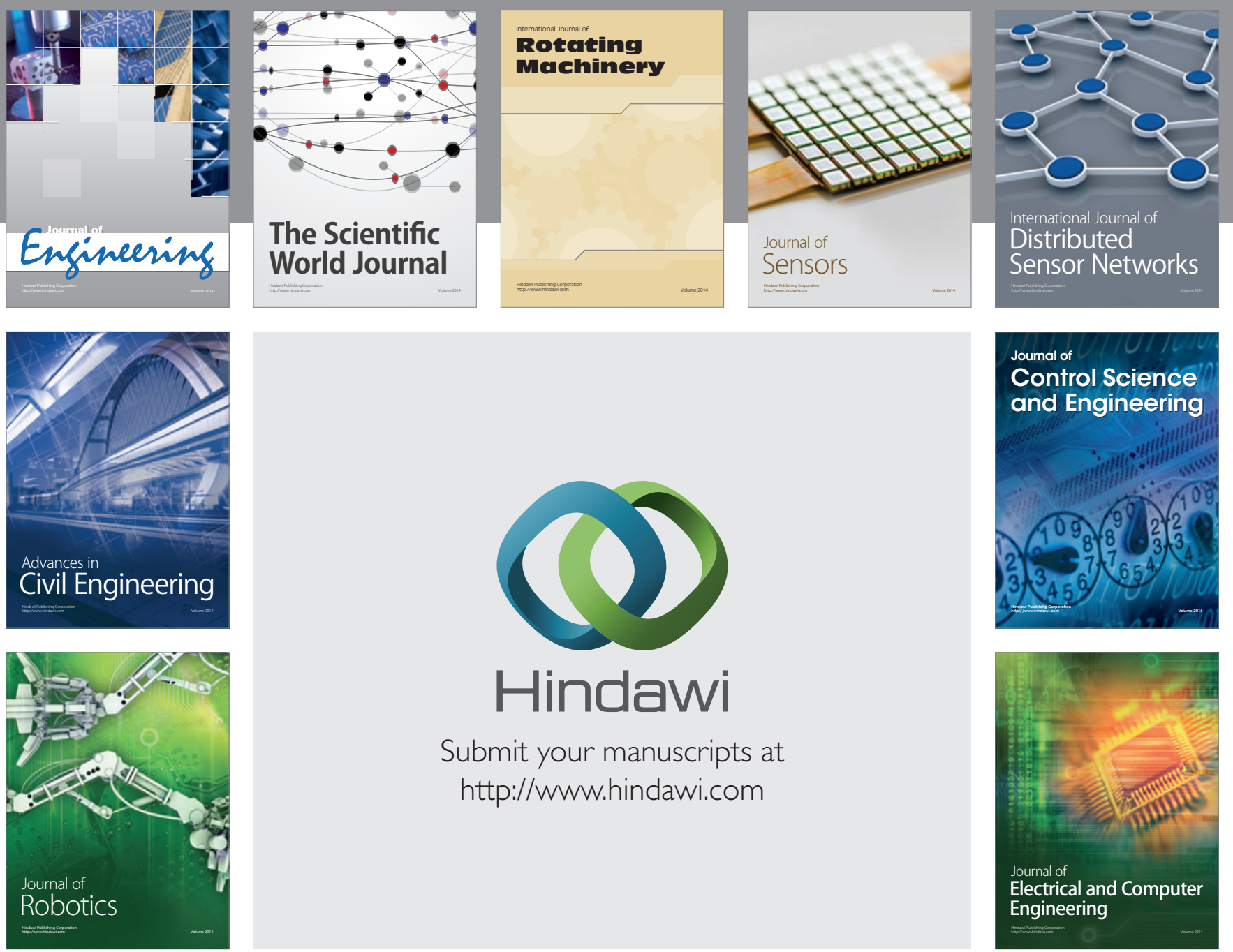

Submit your manuscripts at

http://www.hindawi.com
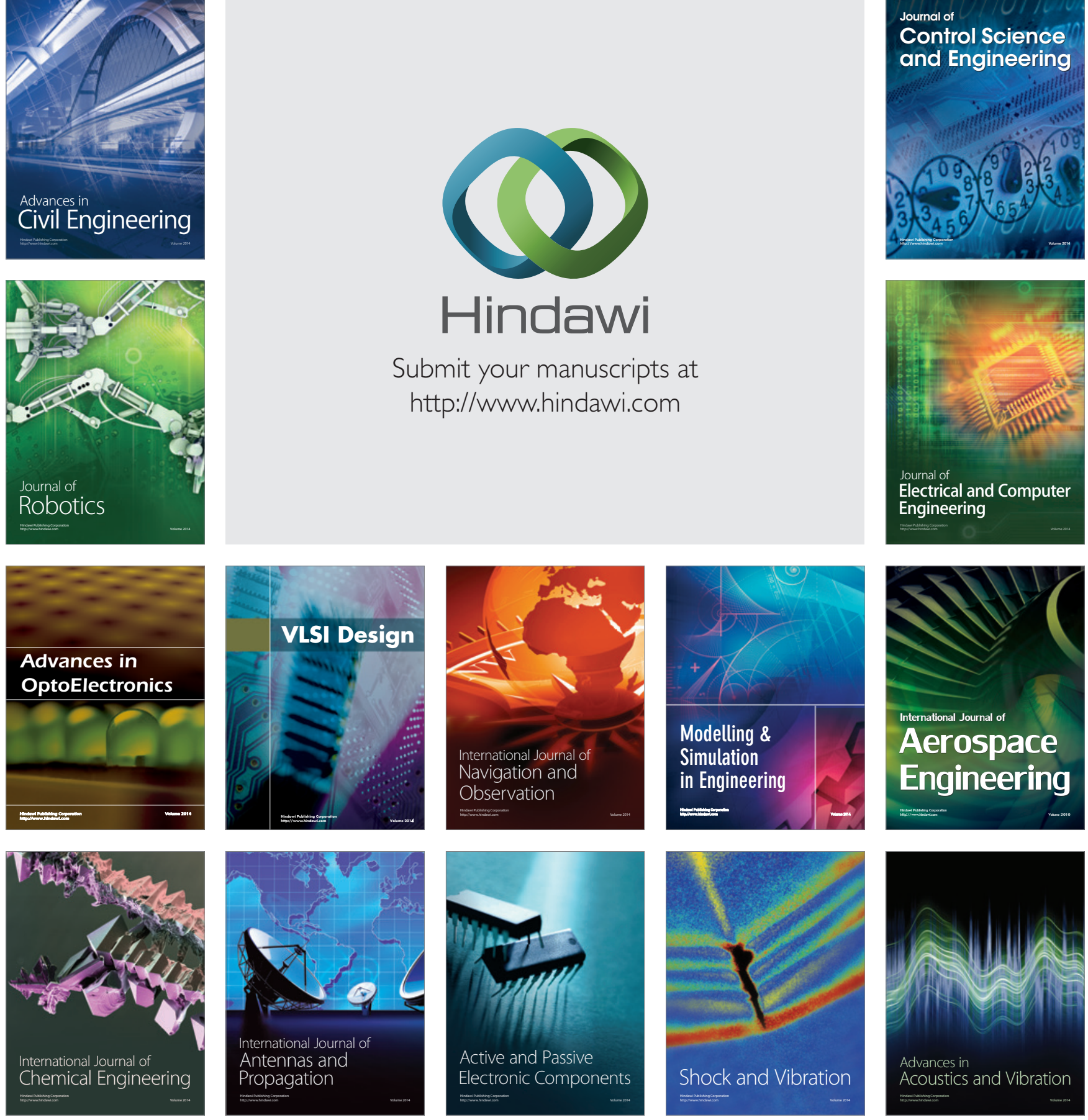